\title{
Tecnura
}

\section{Técnicas de ultrasonido para la caracterización de vidrios volcánicos}

\section{Ultrasonic techniques applied to the characterization of volcanic glass}

\author{
Diego Alejandro Cowes ${ }^{1}$, María Laura Salgán², Juan Ignacio Mieza3, Martín Pedro Gómez
}

Fecha de recepción: 3 de octubre de 2018

Fecha de aceptación: 14 de febrero de 2019

Cómo citar: Cowes, D.A., Salgán, M.L., Mieza, J.I. y Gómez, M.P. (2019). Técnicas de ultrasonido para la caracterización de vidrios volcánicos. Tecnura, 23(60), 27-37. DOI: https://doi.org/10.14483/22487638.14928

\section{Resumen}

Contexto: La caracterización geoquímica de materiales arqueológicos de obsidiana o vidrio volcánico, utilizado en el pasado para la confección de herramientas líticas, permite trazar su procedencia geológica e inferir formas de interacción y rangos de movilidad de antiguas poblaciones humanas. La caracterización de la composición geoquímica de la obsidiana por análisis de activación neutrónica permite determinar con un buen grado de certidumbre su procedencia; sin embargo, es una técnica costosa y destructiva, por lo que no es recomendable para piezas arqueológicas. Otras técnicas más accesibles como la fluorescencia de rayos $X$ o la espectrometría de masas con plasma acoplado inductivamente han sido utilizadas con éxito para esta aplicación pero con un mayor nivel de incertidumbre. En este trabajo se busca asistir al problema por medio del uso de otras técnicas no destructivas que pudieran ser aplicables al patrimonio arqueológico.
Método: Se exploraron las propiedades mecánicas de una muestra de obsidiana a través de métodos de ultrasonido. Se evaluaron la velocidad de fase y atenuación de ondas longitudinales, y se cuantificó la densidad de las muestras por picnometría de gases, que posibilita determinar los módulos elásticos dinámicos. Además, se repitieron las mismas mediciones en probetas de vidrio de manufactura industrial. Resultados: Se observó que la velocidad, la densidad y el módulo longitudinal son mayores para la muestra de vidrio industrial que para la muestra de obsidiana. Por otro lado, la atenuación resultó ser mayor para el caso de la obsidiana y se observó una mayor dependencia con la frecuencia.

Conclusiones: Los resultados obtenidos demuestran la capacidad del método para distinguir entre materiales con composición química diferente, por lo que sería viable para ser aplicado a estudios arqueológicos. Además, las diferencias observadas en la atenuación muestran que el método también es

Ingeniero de sonido, magíster en Ciencia y Tecnología de los Materiales. Comisión Nacional de Energía Atómica. Docente de la Universidad Nacional de Tres de Febrero. Ciudad Autónoma de Buenos Aires, Argentina. Contacto: diegocowes@cnea.gov.ar ORCID: https://orcid. org/0000-0001-6051-4335

2 Licenciada en Antropología, doctora en Ciencias Naturales. Consejo Nacional de Investigaciones Científicas y Técnicas - Instituto de Evolución, Ecología Histórica y Ambiente, Universidad Tecnológica Nacional, Facultad Regional de San Rafael. Docente de IEF. Malargüe, Mendoza, Argentina. Contacto: Isalgan@mendoza-conicet.gob.ar ORCID: https://orcid.org/0000-0002-4741-0280

3 Ingeniero electromecánico, magíster en Ciencia y Tecnología de los Materiales, doctor en Ciencia y Tecnología de los Materiales. Comisión Nacional de Energía Atómica. Docente de la Universidad Nacional de Tres de Febrero y de la Universidad Nacional de San Martín. Ciudad Autónoma de Buenos Aires, Argentina. Contacto: mieza@cnea.gov.ar ORCID: https://orcid.org/0000-0002-4082-7251

4 Licenciado en Física, magíster en Ciencia y Tecnología de los Materiales, doctor en Ciencia y Tecnología de los Materiales. Comisión Nacional de Energía Atómica. Docente de la Universidad Tecnológica Nacional Regional Delta y de la Universidad Nacional de San Martín. Ciudad Autónoma de Buenos Aires, Argentina. Contacto: mpgomez@ cnea.gov.ar ORCID: http://orcid.org/0000-0001-9578-2399 
sensible a la microestructura del material, por lo que aportaría información no accesible mediante técnicas convencionales de arqueometría.

Palabras Clave: arqueología, obsidiana, picnometría, procedencia, pulso-eco, ultrasonido.

\section{Abstract}

Context: The geochemical characterization of archeological materials of obsidian or volcanic glass (used in the past for the preparation of lithic tools) allows to trace origin and infer forms of interaction and ranges of mobility of ancient human populations. The characterization of the geochemical composition of obsidian by analysis of Neutron Activation allows to determine with a good degree of certainty its origin; however, it is a costly and destructive technique, not recommended for archeological pieces. Other more accessible techniques, such as X Ray Fluorescence or Mass Spectrometry with inductively coupled plasma have been used successfully for this application but with a higher level of uncertainty. This work seeks to provide solutions through the use other non-destructive techniques that may be applicable to archeological heritage.
Method: The mechanical properties of an obsidian sample were explored through ultrasound methods. The phase velocity and the attenuation of longitudinal waves were measured, and the density of the samples was quantified with gas pycnometry, which makes it possible to determine the dynamic elastic modules. Additionally, the same measurements were repeated on glass samples of industrial manufacture.

Results: It was observed that the speed, density, and longitudinal modulus were higher for the industrial glass sample than for the obsidian sample. Conversely, the attenuation was greater in the case of obsidian, and a greater dependence on frequency was observed.

Conclusions: The results obtained demonstrate the ability of the method to distinguish between materials with different chemical composition; thus, it would be useful in archeological studies. Moreover, the differences observed in the attenuation show that the method is sensitive to the microstructure of the material, so it would provide information not accessible by conventional archaeometry techniques.

Keywords: Archeology, Obsidian, Pycnometry, Provenance, Pulse-echo, Ultrasound.

\section{INTRODUCCIÓN}

Las herramientas de piedra constituyen el recurso natural más utilizado en la historia de los grupos humanos para realizar tareas básicas, como cazar, cortar, raspar, desbastar, y organizar refugios, entre otros (Odell, 2004). Desde el momento de la fabricación, uso y descarte de la herramienta, hasta su posterior hallazgo y estudio por la arqueología, la mayoría de las propiedades físicas y químicas de las materias primas se mantienen invariantes a los procesos tafonómicos. El estudio de las materias primas brinda valiosa información para establecer la procedencia geológica de la roca y su abundancia en el paisaje, en algunos casos con un buen grado de certeza. En el caso de la obsidiana, su abastecimiento para la manufactura de herramientas, se dio de distintas maneras, desde su recolección local en los ambientes recurrentemente ocupados por los grupos humanos, hasta la utilización de sistemas complejos de comercio e interacción que implicaban traslados y distancias de cientos de kilómetros entre los yacimientos arqueológicos y sus fuentes naturales. Por esto, la distribución geográfica de las piezas arqueológicas confeccionadas en obsidiana y el estudio actual de su procedencia permiten conocer diferentes formas de movilidad, grados de interacción y comercio entre las poblaciones humanas en el pasado (Odell, 2004; Shackey, 1998).

La obsidiana puede categorizarse como un vidrio natural de origen ígneo y textura vítrea y no cristalina (Tarbuck y Lutgens, 2005). Es un sólido amorfo que se forma cuando el magma altamente 
viscoso posee alto contenido de silicio y aluminio, se enfría rápidamente, y el proceso de cristalización se ve obstruido (Glascock, 2002). Generalmente su color es negro, gris, marrón o rojizo, con una tonalidad bandeada. Debido a su estructura atómica desordenada, es físicamente amorfa e isotrópica, de fractura concoide. Esta es una de las principales razones por las que sirve de materia prima para hacer herramientas, ya que al carecer de orientaciones privilegiadas para su corte, se puede fragmentar en diferentes direcciones, generando un borde tenaz y cortante. Algunos tipos de obsidiana presentan inclusiones cristalinas que perjudican sus propiedades de fractura produciendo herramientas de baja calidad.

La composición típica de las obsidianas es de 66-75\% $\mathrm{SiO}_{2}, 10-15 \% \mathrm{Al} 203,3-5 \% \mathrm{Na}_{2} \mathrm{O}$, $2-5 \% \mathrm{~K}_{2} \mathrm{O}$ y $1-5 \% \mathrm{Fe}_{2} \mathrm{O}_{3}+\mathrm{FeO}$ y elementos traza con concentraciones menores al $1 \%$ (Glascock, 2002). La mayoría de las fuentes de obsidiana son químicamente homogéneas con variaciones del orden del $1 \%$ o menores. Sin embargo, diferentes fuentes tienen distintas composiciones como consecuencia de las rocas madre que fueron fundidas y por cambios particulares de cada caso que se producen en el magma antes de la erupción. Aunque los elementos principales en la obsidiana están restringidos a un rango acotado de composiciones, la abundancia de elementos traza puede diferir en órdenes de magnitud entre distintas fuentes. Si la variación entre fuentes es mayor que la variación dentro de una fuente, la procedencia de la obsidiana utilizada en las piezas puede ser establecida con éxito.

Las técnicas de fluorescencia de rayos $X(X R F)$ y análisis por activación neutrónica (NAA) han sido las más utilizadas por la arqueometría. Para el análisis por activación neutrónica, las principales ventajas residen en que los neutrones térmicos son penetrantes y además permite la medición de alrededor de 30 elementos con buena precisión $(<2,5 \%)$. La principal desventaja del método consiste en que una porción de la muestra es destruida, lo cual es indeseable si se trabaja con patrimonio arqueológico. Además, este método requiere un reactor nuclear, por lo que es costoso. La fluorescencia de rayos $X$ tiene la ventaja de que requiere relativamente poca preparación de la muestra, y la destrucción del artefacto es evitada. El análisis es rápido, puede realizarse in situ y el costo es bajo (Glascock, 2002; Salgán, Gil y Neme, 2014; Salgán et al., 2015). Sin embargo, solo se pueden medir en el orden de 10 elementos, la superficie de medición debe ser plana, y puede entregar resultados inconclusos si la muestra es pequeña, delgada o de forma irregular. A su vez, la técnica solo analiza la superficie del material. De esta manera es posible cometer errores al asignar piezas arqueológicas a fuentes líticas.

En este contexto sería de utilidad contar con otras técnicas no destructivas, y de bajo costo que pudieran aportar nuevas variables para disminuir la incertidumbre al asignar fuentes de origen a artefactos arqueológicos de obsidiana. En particular, las ondas elásticas permiten determinar las constantes de rigidez de los sólidos, ya que la velocidad de propagación de aquellas está dada por las propiedades mecánicas. El método ultrasónico de pulso eco se ha utilizado con éxito para estudiar la influencia de la composición química sobre las constantes elásticas dinámicas en vidrio industrial (cuya composición es similar a la obsidiana) observándose buena correlación entre dichas variables (Kilinc, 2016). Por otro lado, el estudio de la atenuación ultrasónica en vidrio ha mostrado picos de absorción cuyas características y posición dependen de la composición del vidrio y la frecuencia de la onda aplicada (Sidkey et al., 1997).

Tsobgou y Dabard (2010) utilizaron la técnica de ultrasonido como parte fundamental del análisis estadístico multivariado para la clasificación de piedras de acuerdo con sus propiedades estructurales y mineralógicas. A partir de esto obtuvieron la distribución regional de la materia prima lítica utilizada por grupos humanos durante el periodo Mesolítico y Neolítico en el macizo Armoricano (Tsobgou, 2009). Además, se evaluó la influencia de los parámetros mecánicos en la selección, 
fabricación y uso de las herramientas de piedra prehistóricas en busca de información acerca de la elección de los tipos de herramientas, evolución tecnológica y la distribución territorial de grupos humanos (Tsobgou y Dabard, 2010). Se observó que las principales propiedades que se correlacionan con la elección de materiales son la isotropía y la fragilidad, en el caso del Mesolítico, y dureza del cuerpo de la herramienta y del filo, durante el Neolítico.

En otros ejemplos se empleó ultrasonido como método complementario a ensayos de fractomecánica en obsidiana. En particular se estudiaron el comportamiento de microfractura (Husien, 2004), la dureza del filo (McPherron et al., 2014), y la elasticidad e isotropía (Prost y Chatellier, 1986). De esta manera se pudo caracterizar la calidad de las distintas fuentes en función de su capacidad de producir herramientas. Sin embargo, la fractomecánica implica la destrucción de la muestra por lo que es aplicable a la caracterización de fuentes, pero no a piezas arqueológicas.

El ultrasonido también ha sido utilizado por la geofísica y geología para caracterizar obsidianas, ya que este dato es útil para desarrollar modelos de propagación de ondas sísmicas (Woeber, Katz y Ahrens, 1963; Suito, Miyoshi, Sasakura, Fujisawa, 1992; Anderson y Schreiber, 1996; Sato, Ito y Aizawa, 2004). Si bien esta disciplina no aporta conocimiento de carácter antropológico, constituye un punto para comparación de resultados.

En este trabajo se analizó la viabilidad de técnicas ultrasónicas con el fin de aportar nuevas variables para disminuir la incertidumbre al asignar artefactos de obsidiana a las fuentes de origen, ya que son de naturaleza no destructiva y son útiles para caracterizar el comportamiento elástico mecánico de los materiales. Técnicas que no cuentan con antecedentes para la arqueología argentina. Para esto se abordaron ensayos preliminares en muestras de obsidiana y de vidrio común para establecer si el método es lo suficientemente sensible como para distinguir entre dichos materiales.

\section{MARCO TEÓRICO}

\section{Constantes elásticas}

Las propiedades mecánicas de un material se pueden caracterizar a través de su tensor de constantes elásticas $C_{i j k \prime}$ el cual relaciona la tensión y la deformación en régimen lineal y se expresa a través de la ley de Hooke generalizada, ecuación (1) (Cheeke, 2012).

$$
\sigma_{i j}=C_{i j k l} \varepsilon_{k l}
$$

El mismo tiene 81 valores los cuales, por simetría en los tensores de tensión y deformación, se reducen a 36. La condición de preservación de la energía de deformación reduce dicho tensor a 21 constantes. En el caso de que el sólido tenga las mismas propiedades independientemente de la dirección (isotropía lineal) implica que dichas constantes se pueden reducir a solo 2 independientes. A partir de estas se definen otros parámetros como el primer parámetro de Lamé I (ver ecuación (2)), el módulo de elasticidad transversal m o $G$ (ver ecuación (3)), el módulo de compresibilidad $K$, el módulo de Young $E$, el módulo de elasticidad longitudinal $M, y$ el coeficiente de Poisson $\mathrm{n}$. Entre estos, dos parámetros cualesquiera describen completamente las propiedades elásticas de los sólidos linealmente isotrópicos como se muestra en la ecuación (4).

$$
\begin{gathered}
C_{11}=\lambda+2 \mu \\
C_{12}=\lambda \\
\sigma=2 \mu \varepsilon+\lambda \operatorname{tr}(\varepsilon) I
\end{gathered}
$$

Donde / es la matriz identidad y $\operatorname{tr}($ ) es la función traza. 


\section{Ondas elásticas}

Existen distintos tipos de técnicas para medir las propiedades elásticas de un sólido, y las podemos clasificar en estáticas y dinámicas. Los ensayos estáticos consisten en medir directamente la fuerza aplicada y la deformación que se produce durante pruebas mecánicas. Entre estos métodos se encuentran la flexión de cuatro puntos y la nanoindentación. Las técnicas dinámicas presentan la ventaja que pueden utilizar una mayor variedad de tipos de muestras en tamaño y forma, con gran precisión y en un amplio rango de temperatura. Entre los métodos dinámicos se destacan los de resonancia y los de pulsos. Estos últimos consisten en introducir una perturbación en el material y a partir del tiempo que tarda en recorrer su espesor, se determina la velocidad de onda y al relacionarla con la densidad permite determinar módulos elásticos. En este trabajo se utilizaron métodos dinámicos de pulsos ultrasónicos por su naturaleza no destructiva.

La ecuación general de onda para el movimiento de una partícula de material perfectamente elástico, de tamaño infinitesimal y para desplazamientos de primer orden está dada por la ecuación (5).

$$
\rho \frac{\partial^{2} u}{\partial t^{2}}=C \frac{\partial^{2} u}{\partial x^{2}}
$$

En el interior de un sólido isotrópico se pueden propagar dos tipos de ondas acústicas: longitudinales (P) y transversales (S). La onda longitudinal es una perturbación ocasionada por una tensión normal, donde el desplazamiento de las partículas del material ocurre en la misma dirección de propagación de la perturbación. Las ondas transversales son ocasionadas por tensiones de cizallamiento y el desplazamiento de las partículas ocurre en el plano perpendicular a la propagación. A partir de la ecuación (5) es posible relacionar las velocidades de los modos $\mathrm{P}$ y $\mathrm{S}$ con las constantes elásticas como se muestra en las ecuaciones (6) y (7).

$$
\begin{aligned}
& M=\rho V_{l}^{2} \\
& \mu=\rho V_{s}^{2}
\end{aligned}
$$

De esta forma a partir de la velocidad de ondas longitudinales, la velocidad de ondas transversales y la densidad se caracteriza el comportamiento elástico de un sólido isotrópico.

\section{METODOLOGÍA}

\section{Pulso eco}

Para determinar la velocidad del sonido se implementó la técnica de pulso-eco que consiste en colocar un transductor piezoeléctrico sobre el material, e introducir una perturbación mecánica que se propaga en forma de onda. Esta onda viaja hasta la cara opuesta de la pieza (caras planas) donde se refleja y vuelve hacia el transductor que lo transforma en señal eléctrica. En la figura 1 se incluye un diagrama de la medición y en la figura 2 se observa un tren de ecos obtenido por esta técnica. Otra técnica viable para este tipo de ensayos es el ultrasonido por transmisión (Quintero Ortiz, Cruz-Hernández y Peña-Santos, 2014).

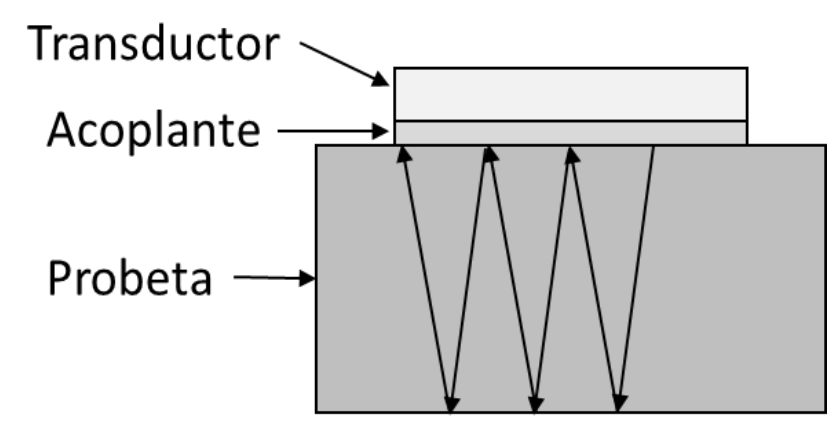

Figura 1. Esquema de medición de pulso-eco

Fuente: elaboración propia. 


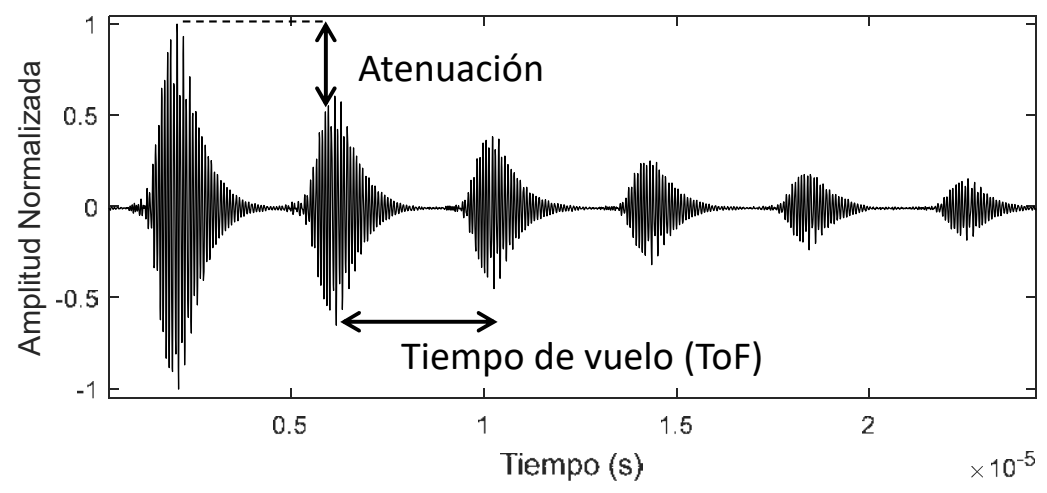

Figura 2. Tren de ecos obtenido por pulso-eco

Fuente: elaboración propia.

A partir de la diferencia de tiempo que hay entre dos ecos sucesivos (time of flight) y el espesor de la probeta, se calcula la velocidad como se muestra en la ecuación (8); y a partir del decaimiento relativo se calcula el coeficiente de atenuación representado en la exponencial decreciente de la solución de la ecuación de onda (ver ecuación (9)).

$$
\begin{gathered}
\mathrm{V}=\frac{2 \mathrm{~d}}{\mathrm{ToF}} \\
A(z, t)=A_{0} e^{-\alpha z} e^{i(\omega t-k z)}
\end{gathered}
$$

Donde, $A_{0}$ es la amplitud inicial, $\omega$ es la frecuencia angular, $\alpha$ es el coeficiente de atenuación, $t$ es el tiempo, $k$ es el número de onda y $z$ es la posición.

Más detalles del método utilizado pueden encontrarse en Cowes (2018) y Papadakis (1973).

\section{Picnometría}

La picnometría de gases es una de las técnicas más precisas para medir volumen a partir del principio de desplazamiento de Arquímedes y la ley de Boyle de expansión de gases. En lugar de líquido, se emplea un gas inerte ya que penetra en los poros más pequeños y elimina la influencia de fenómenos químicos y físicos superficiales. El helio es el gas inerte utilizado por excelencia. La técnica consiste en introducir la muestra en un recipiente de volumen conocido para luego ser presurizado a una presión determinada. Una vez que la presión se estabiliza y se registra, se abre una válvula que permite la expansión del gas hacia otro volumen conocido. Cuando se estabiliza el sistema, se calcula la caída de presión y a partir de esta se obtiene el volumen de la muestra.

\section{Preparación de muestras}

Se preparó una probeta de obsidiana a partir de un fragmento natural obtenido de la fuente laguna del Maule, ubicada próxima al límite internacional entre Argentina y Chile, en la cordillera de los Andes. Los estudios arqueológicos realizados hasta el momento dan cuenta de que la fuente laguna del Maule fue utilizada en ambos flancos de los Andes, en los últimos ca. 8000 años antes del presente (a.p.) (Giesso et al., 2011; Salgán, Gil y Neme, 2012). Su composición química se fue caracterizada por XRF e NAA como parte de diferentes proyectos de investigación (Seelenfreud et al., 1996; Neme y Gil, 2008; Giesso et al., 2011; Cortegoso et al., 2012). Para llevar adelante el presente ensayo, se realizó un corte con sierra diamantada, y se pulió a caras paralelas mediante un tornillo de pulido para tal fin. Se obtuvo una muestra a caras 
paralelas con bordes irregulares, pero las dimensiones son tales que la onda no interactúa con dichos bordes. A su vez, se obtuvieron muestras de vidrio común de dos espesores distintos, 6 y 10 $\mathrm{mm}$, para evaluar si la técnica es capaz de diferenciar entre los materiales. El vidrio no fue pulido ya que el acabado superficial y el paralelismo eran suficientemente buenos para los ensayos realizados. Las probetas se muestran en la figura 3.

En la tabla 1 se incluye la comparación de composición de óxidos entre vidrio común y dos tipos de obsidiana. Estos datos son de referencia ya que la composición de la obsidiana utilizada en este trabajo está expresada en elementos y no resulta idónea para este fin.

\section{Mediciones}

El espesor de las muestras fue obtenido por un micrómetro digital calibrado cuya incertidumbre es de $+/-1 \mathrm{~mm}$. Luego se obtuvo la densidad midiendo la masa en una balanza de dos dígitos decimales y el volumen en el picnómetro de gases de alta precisión micromeritics AccuPyc II 1340 con helio. Por último, se realizaron los ensayos de pulso-eco con el equipo generador y amplificador de pulsos Matec 7700 y el inserto 760v con el sensor Matec AD-30c a una frecuencia central de $20 \mathrm{MHz}$. Las señales fueron acondicionadas y digitalizadas por un oscilocopio digital PicoScope 4227 y posteriormente fueron procesadas en MATLAB.

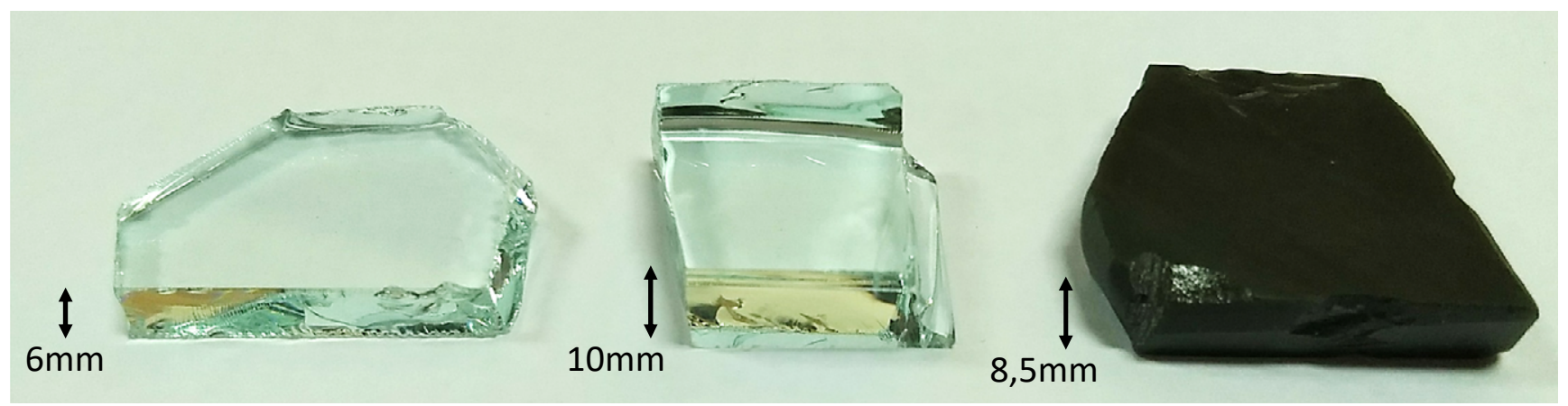

Figura 3. Probetas. De izquierda a derecha: vidrio delgado, vidrio grueso, obsidiana de laguna del Maule

Fuente: elaboración propia.

Tabla 1. Composición en óxidos de vidrio y obsidianas de referencia

\begin{tabular}{lccccccc}
\hline Composición de óxidos (\% en peso) & $\mathrm{SiO}_{2}$ & $\mathrm{Na}_{2} \mathrm{O}$ & $\mathrm{CaO}$ & $\mathrm{Al}_{2} \mathrm{O}_{3}$ & $\mathrm{~K}_{2} \mathrm{O}$ & $\mathrm{Fe}_{2} \mathrm{O}_{3}$ & $\mathrm{MgO}$ \\
\hline Vidrio (Bretschneider y Drexler, 2001) & $66-75$ & $10-20$ & $5-15$ & $0-5$ & $0-5$ & $\leqq 0.03$ & $0-6$ \\
\hline Obsidiana, Nevada, EE. UU. (Husien, 2004) & 79,3 & 4,1 & 0,4 & 12,4 & 4,0 & 1,5 & 0,1 \\
\hline Obsidiana, Wada Togue, Japón (Suito et al., 1992) & 76,2 & 2,8 & 1,0 & 12,6 & 4,2 & 0,7 & 0,2 \\
\hline
\end{tabular}

Fuente: elaboración propia.

\section{RESULTADOS Y DISCUSIÓN}

En la tabla 2 se incluyen los resultados de las mediciones. El espesor, el tiempo de vuelo, la masa y el volumen son magnitudes medidas, mientras que la velocidad longitudinal, la densidad y el módulo longitudinal son magnitudes calculadas y se diferencian en negrita. Estas últimas, al ser propiedades intensivas permiten caracterizar al material y pueden ser utilizadas para distinguir entre materiales. Se observa que, dentro de los intervalos de confianza, las magnitudes obtenidas para ambos vidrios se solapan, es decir que son equivalentes. Para todos los parámetros, la muestra delgada 
presenta mayor incertidumbre que la gruesa, es decir que la última tiene una geometría más favorable para este tipo de ensayos. Por otro lado, la muestra de obsidiana, siendo vidrio de otra composición, presenta valores menores en todos los parámetros intensivos, aun considerando la incertidumbre. A partir de esto se deduce que el método sería útil para distinguir entre tipos de amorfos cuando la variación de composición es considerable. A partir de esto, se evidencian dos líneas de acción para la continuación de este trabajo. En primer lugar, tanto tensiones residuales como anisotropía de solidificación podrían variar las propiedades mecánicas dentro una misma fuente, por lo que se requiere realizar un mayor número de mediciones para establecer la variabilidad de parámetros dentro de una misma fuente. Por otro lado, la variación de composición entre obsidianas de distintas fuentes se estima que es menor que entre vidrio y obsidiana de acuerdo a lo presentado en la tabla 1, por ende, debe repetirse este proceso para distintas fuentes. Si la variabilidad dentro de una fuente es menor que entre fuentes entonces los parámetros medidos podrían ser útiles para determinar la procedencia de artefactos líticos de origen arqueológico. Por otro lado, sería útil incluir mediciones con ondas transversales para aportar un mayor número de parámetros y lograr una mejor caracterización mecánica. Posteriormente los parámetros podrían ser estudiados por análisis de componentes principales con el fin de reducir la dimensionalidad del conjunto de datos (Rueda-Bayonal et al., 2016).

La atenuación de ondas longitudinales se presenta en la figura 4. En primera instancia se observa que dentro del intervalo de confianza del método (Cowes, 2018) la atenuación para las probetas de vidrio se solaparía, por lo que es indistinta. Por otro lado, en el rango de frecuencias de medición, se observa una mayor atenuación para la obsidiana que para los vidrios, probablemente debido a la presencia de dispersores acústicos constituidos por discontinuidades microestructurales. A su vez la dependencia de la atenuación con la frecuencia es mayor para la obsidiana que para los vidrios y esto puede estar en relación con el tamaño de los dispersores y la longitud de onda utilizada. Este parámetro puede resultar útil para determinar la procedencia de obsidianas, ya que la atenuación dominada por la microestructura puede ser independiente de la composición química, pero aun así característica de una fuente en particular. Sería pertinente contrastar estos resultados con análisis micrográficos de los mineraloides; también, sería viable incluir análisis por elementos finitos para simular la propagación en el sólido (Ramos-Cañón, Prada-Sarmiento y Vega-Posada, 2016).

Tabla 2. Resultados de las mediciones

\begin{tabular}{lllllll}
\hline \multicolumn{1}{c}{ Muestra } & \multicolumn{2}{c}{$\begin{array}{c}\text { Vidrio común } \\
\text { delgado }\end{array}$} & \multicolumn{2}{c}{$\begin{array}{c}\text { Vidrio común } \\
\text { grueso }\end{array}$} & \multicolumn{2}{c}{$\begin{array}{c}\text { Obsidiana (laguna } \\
\text { del Maule) }\end{array}$} \\
\hline Espesor $[\boldsymbol{\mu m}]$ & 5874 & $(1)$ & 9882 & $(1)$ & 8502 & $(1)$ \\
\hline Tiempo de vuelo $[\boldsymbol{\mu s}]$ & 2,020 & $(, 001)$ & 3,397 & $(, 001)$ & 2,941 & $(, 001)$ \\
\hline Velocidad longitudinal $[\mathbf{m} / \mathbf{s}]$ & 5816 & $(3,0)$ & 5817 & $(1,8)$ & 5782 & $(2,1)$ \\
\hline Masa $[\mathbf{g}]$ & 7,87 & $(, 01)$ & 10,46 & $(, 01)$ & 13,13 & $(, 01)$ \\
\hline Volumen $\left[\mathbf{c m}^{\mathbf{3}}\right]$ & 3,158 & $(, 008)$ & 4,184 & $(, 004)$ & 5,545 & $(, 002)$ \\
\hline Densidad $\left[\mathbf{g} / \mathbf{c m}^{\mathbf{3}}\right]$ & 2,492 & $(, 007)$ & 2,500 & $(, 004)$ & 2,368 & $(, 002)$ \\
\hline Módulo longitudinal $\mathbf{M}[\mathbf{G p a}]$ & 84,3 & $(, 3)$ & 84,6 & $(, 1)$ & 79,2 & $(, 1)$ \\
\hline
\end{tabular}

Fuente: elaboración propia. 


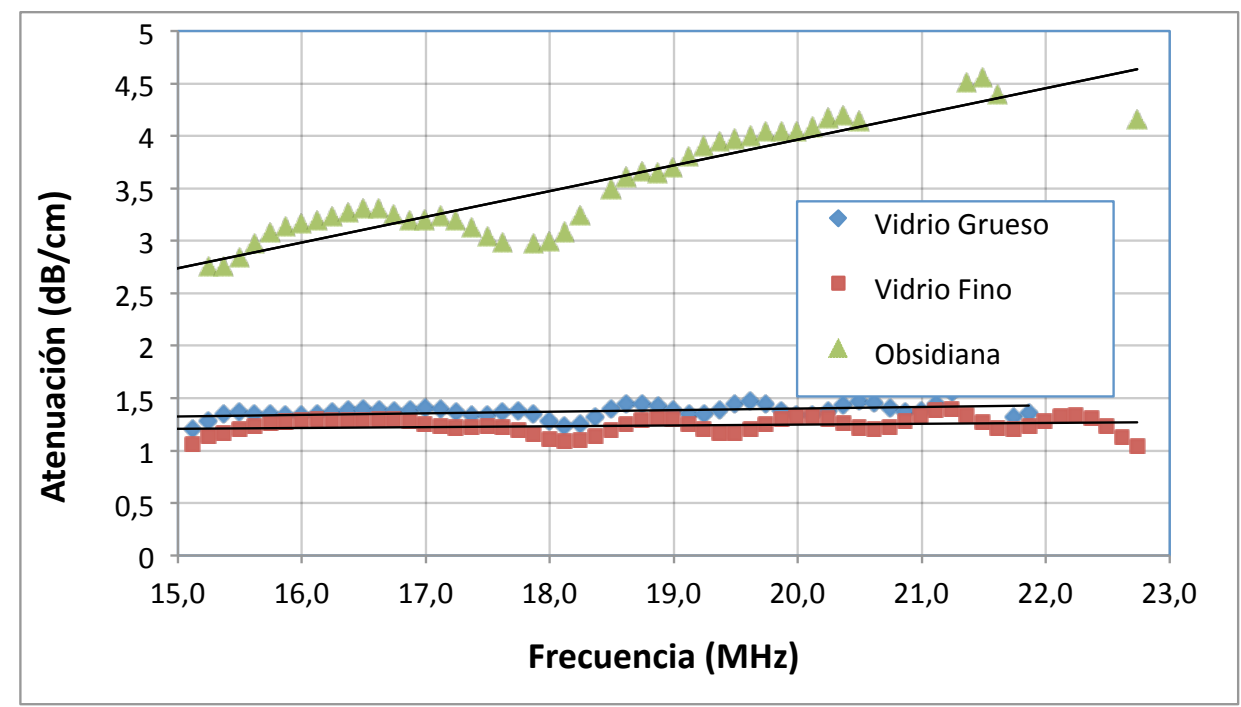

Figura 4. Atenuación de ondas longitudinales en función de la frecuencia

Fuente: elaboración propia.

\section{CONCLUSIÓN}

En el presente trabajo se realizaron mediciones en una muestra natural de obsidiana con el fin de caracterizar su comportamiento mecánico elástico y de aportar variables a los estudios de procedencia de bienes arqueológicos. Se realizaron mediciones de ultrasonido por pulso-eco con ondas longitudinales a partir de las cuales se obtuvieron el tiempo de vuelo y la atenuación. A su vez, se midieron el espesor y la masa de las probetas, y se determinó el volumen por la técnica de picnometría de gases de alta precisión. Todas las mediciones también se realizaron sobre muestras de vidrio para establecer la capacidad del método de distinguir entre estos amorfos con diferente composición química. Se observó que los parámetros obtenidos presentan diferencias significativas entre los diferentes materiales por lo que se concluye que el método logra dicha distinción. Para continuar esta línea de investigación es necesario caracterizar la variabilidad dentro de una misma fuente y posteriormente entre fuentes. Además, se observó que la atenuación para obsidianas resulta mayor que para el vidrio, y se estima que esto responde a características microsetructurales que no son directamente dependientes de la composición química. Por tanto, este parámetro podría aportar información novedosa, no accesible mediante técnicas geoquímicas, y complementaria para los estudios de procedencia arqueológicos o de autenticidad de piezas de museos. Por otro lado, sería útil incluir mediciones con ondas transversales para acceder a un mayor número de parámetros. A su vez, si se corrobora la capacidad del método de distinguir entre fuentes, será necesario adaptarlo para ser aplicado en piezas arqueológicas que, por lo general, no presentan caras paralelas. Esto podría lograrse a través de la focalización del haz u otra técnica análoga.

\section{FINANCIAMIENTO}

Investigación asociada al proyecto International Centre of Earth Sciences (ICES), y fue avalada y financiada por la Comisión Nacional de Energía Atómica.

Parte de esta investigación fue realizada gracias a los fondos aportados por Conicet y la Agencia Nacional de Promoción Científica y Tecnológica de Argentina (PICT 2014-3270). 


\section{REFERENCIAS}

Anderson, O.L. y Schreiber, E. (1966). Measurement of $P$ and S Sound Velocities Under Pressure on Laboratory Models of the Earth's Mantle. Annual Summary Report. Palisades, Nueva York: Lamont Geological Observatory, Columbia University.

Bretschneider, J. y Drexler, H. (2001). Soda-lime-silicate glass composition. Patente US6218323B1. Estados Unidos.

Cheeke, J.D.N. (2012). Fundamentals and Applications of Ultrasonic Waves. Boca Ratón: CRC Press.

Cortegoso, V., Neme, G., Giesso, M., Durán, V. y Gil, A. (2012). El uso de la obsidiana en el sur de Mendoza. En A. Gil y G. Neme (eds.), Paleoecología humana en el sur de Mendoza (pp. 180-211). Buenos Aires: Sociedad Argentina de Antropología.

https://doi.org/10.4067/s0718-10432004002800004

Cowes, D.A. (2018). Técnicas de ultrasonido para la determinación del contenido de $\mathrm{H}$ en aleaciones de Zr. [Tesis de maestría]. Maestría en Ciencia y Tecnología de Materiales, Instituto Sabato, UNSAM, Argentina.

https://doi.org/10.17227/ted.num28-1079

Giesso, M., Durán, V., Neme, G., Glascock, M.D., Cortegoso, V., Gil, A. y Sanhuesa, L. (2011). A study of obsidian source usage in the central Andes of Argentina and Chile. Archaeometry, 53(1), 1-21.

https://doi.org/10.1111/j.1475-4754.2010.00555.x

Glascock, M.D. (2002). Obsidian provenance research in the Americas. Accounts of Chemical Research, 35(8), 611-617.

https://doi.org/10.1021/ar010041f

Husien, M.S. (2004). Fracture behavior and mechanical characterization of obsidian: naturally occurring glass. [Bachelor of science thesis]. Bahir Dar University, Ethiopia.

Kilinc, E. (2006). Mechanical and structural properties of soda lime silica glasses as a function of composition. [Ph. D. Thesis]. The University of Sheffield, Faculty of Engineering, Department of Materials Science and Engineering. England.

McPherron, S.P., Braun, D., Dogandžić, T., Archer, W., Desta, D. y Lin, S.C. (2014). An experimental assessment of the influences on edge damage to lithic artifacts: A consideration of edge angle, substrate grain size, raw material properties, and exposed face. Journal of Archaeological Science, 49, 70-82.

https://doi.org/10.1016/j.jas.2014.04.003

Neme, G. y Gil, A. (2008). Biogeografía humana en los Andes Meridionales: tendencias arqueológicas en el sur de Mendoza. Chungará, 40, 5-18.

Odell, G. (2004). Manuals in Archaeological Method, Theory, and Technique. En E. Orser and B. Schiffer (eds.), Lithics Analysis (pp. 175-218). Nueva York: Springer Science+Business Media.

Papadakis, E.P. (1973). The measurement of small changes in ultrasonic velocity and attenuation. CRC Critical Reviews in Solid State Sciences, 3(4), 373-418.

https://doi.org/10.1080/10408437308244869

Prost, D.C. y Chatellier, J. (1986). Contribution méthodologique à I'analyse quantitative des propriétés mécaniques des roches fragiles élastiques. Revue d'Archéométrie, 10, 69-74.

https://doi.org/10.3406/arsci.1986.1228

Quintero-Ortiz, L.A., Cruz-Hernández, R.A. y Peña-Santos, D.Y. (2014). Efecto del contenido de agua sobre la resistencia y la velocidad de pulso ultrasónico del concreto. Tecnura, 18(42), 103-113.

https://doi.org/10.14483/udistrital.jour. tecnura.2014.4.a08

Ramos-Cañón, A.M., Prada-Sarmiento, L.F. y Vega-Posada, C.A. (2016). Análisis de elementos finitos con un continuo elástico lineal tipo Cosserat. Tecnura, 20(50), 43-53.

Rueda-Bayona1, J.G., Elles-Pérez, C.J., Sánchez-Cotte, E.H., González-Ariza, A.L. y Rivillas-Ospina, G.D. (2016). Identificación de patrones de variabilidad climática a partir de análisis de componentes principales, Fourier y clúster k-medias. Tecnura, 20(50), 55-68.

Salgán, M.L., Gil, A. y Neme, G. (2012). Obsidianas en La Payunia (sur de Mendoza, Argentina): patrones de distribución e implicancias en la ocupación regional. Magallania, 40(1), 263-277.

https://doi.org/10.4067/s0718-22442012000100015

Salgán, M.L., Gil, A. y Neme, G. (2014). Movilidad, aprovisionamiento y uso de obsidiana en El Payén, 
sur de la provincia de Mendoza, Argentina. Comechingonia, 18(1), 33-50.

https://doi.org/10.4067/s0718-10432004002800004

Salgán, L., Garvey, R., Neme, G., Gil, A., Giesso, M., Glascock, M.D. y Durán, V. (2015). Las Cargas: Characterization and Prehistoric Use of a Southern Andean Obsidian Source. Geoarchaeology: An International Journal, 0, 1-12.

https://doi.org/10.1002/gea.21502

Sato, H., Ito, K. y Aizawa, Y. (2004). Simultaneous velocity and attenuation measurements applicable to various solids at high pressures and temperatures up to $1200^{\circ}$ C. Meas. Sci. Technol., 15, 1787-1793. https://doi.org/10.1088/0957-0233/15/9/016

Seelenfreund, A., Rees, C., Bird, R., Bailey, G., Bárcena, R. y Durán, V. (1996). Trace element analysis of obsidian sources and artifacts of Central Chile (Maule River Basin) and Western Argentina (Colorado River). Latin American Antiquity, 7, 7-20.

https://doi.org/10.2307/3537011

Sidkey, M.A., Mallawany, R.E., Nakhla, R.I. y El-Moneim, A. Abd. (1997). Ultrasonic Attenuation at Low Temperature of TeO2 -V2O5. Glasses. Phys. Stat. Sol.,159, 397.

https://doi.org/10.1002/1521-396x(199702)159:2<397 ::aid-pssa397>3.0.co;2-0
Suito, K., Miyoshi, M., Sasakura, T. y Fujisawa, H. (1992). Elastic properties of obsidian, vitreous $\mathrm{SiO}_{2}$, and vitreous $\mathrm{GeO}_{2}$ under high pressure up to 6 Gpa. Geophysical Monograph Series, 67, 219-225.

https://doi.org/10.1029/gm067p0219

Tarbuck, E.J. y Lutgens, F.K. (2005). Ciencias de la Tierra: Una introducción a la Geología física. 8a. ed. Madrid: Pearson Prentice Hall.

Tsobgou, R. A. (2009). Mapping Mesolithic and Neolithic cultures behaviours and interactions with nature and properties of rocks in Western France. Journal of Archaeological Science, 36, 1615-1625.

https://doi.org/10.1016/j.jas.2009.03.024

Tsobgou, A.R. y Dabard, M.P. (2010). Petrographical, Structural, and Mechanical Analysis of Armorican Phtanites: A Key Raw Material for the Mesolithic in Western France. Geoarchaeology: An International Journal, 25(3), 327-351.

https://doi.org/10.1002/gea.20310

Woeber, A.F., Katz, S. y Ahrens, T.J. (1963). Elasticity of selected rocks and minerals. Geophysics, 28(4), 658-663.

https://doi.org/10.1190/1.1439242

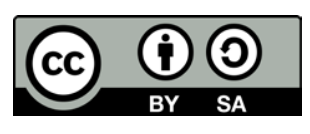

Tecnura • p-ISSN: 0123-921X • e-ISSN: 2248-7638 • Vol. 23 No. $60 \bullet$ Abril - Junio de $2019 \bullet$ pp. 27-37 